\title{
The effect of metastable level populations on the ionization fraction of Li-like ions
}

\author{
J. G. Doyle ${ }^{1, \star}$, H. P. Summers ${ }^{2}$, and P. Bryans ${ }^{2}$ \\ 1 Armagh Observatory, College Hill, Armagh, BT61 9DG, N. Ireland \\ e-mail: jgd@arm.ac.uk \\ 2 Department of Physics, University of Strathclyde, Glasgow, Scotland
}

Received 11 November 2004 / Accepted 11 December 2004

\begin{abstract}
Lines from Li-like ions have been known to produce theoretical intensities under-estimated compared to lines of a similar formation temperature. Here we investigate this anomalous behaviour whereby the ionization fractions are calculated using the ADAS code considering the electron density dependence of dielectronic recombination coupled with collisional ionization from metastable levels. For the lines investigated, the line contribution functions show a clear dependence with increasing electron density. For example, C IV $1548 \AA$ shows over a factor of three enhancement for $N_{\mathrm{e}}=10^{12} \mathrm{~cm}^{-3}$. The increase in the higher temperature lines is lower, but are still in the range of 30 to $60 \%$. Furthermore, all the lines have their peak contribution shifted to lower temperature. Calculating the total radiative power output at an electron density of $10^{11} \mathrm{~cm}^{-3}$, we find that the difference in the transition region is $10-15 \%$ while above $10^{6} \mathrm{~K}$ the difference is around $30 \%$ compared to the low density value.
\end{abstract}

Key words. Sun: atmosphere - di-electronic recombination - metastable levels - contributon function - electron density line intensity - UV/EUV radiation

\section{Introduction}

The analysis of UV and EUV lines is essential for a proper understanding of high temperature plasma, e.g. that found in the upper solar/stellar atmospheres. Data from spectrographs aboard the Solar Heliospheric Observatory (SoHO) has lead to a wealth of observations of small-scale dynamic events observed from the Sun's chromosphere to the transition region and corona. On the stellar side, data from the International Ultraviolet Explorer (IUE), Space Telescope Imaging Spectrograph (STIS/HST) and the Far Ultraviolet Spectroscopic Explorer (FUSE) have all provided high quality data which have been used to diagnose properties of the atmospheres in a range of objects. Interpretation of this data is highly dependent on many atomic physics parameters, one of these being the ionization fractions of the ion under consideration (see Young et al. 2003 for a discussion of some of these within the CHIANTI database).

It is been known for many years that lines from $\mathrm{Li}$-like ions can in some instances give very different results from other isoelectronic sequences. Following on from work by Burgess \& Summers (1969), Vernazza \& Raymond (1979) showed that a significant increase in intensity can occur at high electron densities if one considers electron density dependent dielectronic recombination. In the vast majority of published ionization fractions, the low-density assumption is used, and

\footnotetext{
* http://star.arm.ac.uk/preprints/
}

therefore use of these calculations to produce a differential emission measure (DEM) curve using a range of lines including Li-like ions can produce discrepant results (Doyle \& Raymond 1984; Del Zanna et al. 2003). In many instances, due to the limitation in the number of available spectral lines from IUE data, emission measure curves for stellar atmospheres have been derived based on data from Li-like ions, e.g. C IV 1548/50, $\mathrm{N} v$ 1328/42, etc.

The usual practice when considering atomic processes in high-temperature, low-density plasmas, such as discussed here, is to adopt the coronal approximation. This treats the populations of excited states of ions via an excitation balance of collisional excitation, usually from the ground state, by electrons, and radiative decay. The ionization state is established as a balance of electron impact ionization from the ground state and radiative plus dielectronic recombination. In the simplest version of such modelling, secondary collisions with excited states are neglected.

However, there is no consistent treatment of metastable states with populations comparable to the ground state. Thus, in the coronal limit, ionization and excitation balance are independent of electron density. Here we look again into this problem and in particular construct line contribution functions, and then calculate the percentage increase in the line flux for a range of lines. In addition, we calculate the percentage change in the radiative loss function as a result of changes in the electron density. 


\section{Atomic background}

The present work aims to determine what effect the full density-dependence of the excitation and ionization balance, including the full role of low-lying metastables, has on emission from Li-like ions in the solar corona. The generalized collisional-radiative picture (GCR; Summers \& Hooper 1983), which builds on the collisional-radiative theory of Bates et al. (1962), allows such an analysis. In detail, the collisional ionization and redistribution processes from excited states, ignored in the coronal model, are included. The populated metastable states are determined via an elaborated ionization balance along with the ground states.

If one denotes metastable states (including the ground state) by Greek indices and excited states by Roman indices then the sum of transition rates between states can be represented by the collisional-radiative matrix, $C$. Such a matrix contains elements $C_{\rho \sigma_{+}}$and $C_{i \sigma_{+}}$, denoting the sum of the recombination rates from each metastable; $C_{\rho \sigma_{-}}$and $C_{i \sigma_{-}}$, denoting ionization rates; and diagonal elements $C_{\rho \rho}$ and $C_{i i}$, indicating total loss rates from the levels $\rho$ and $i$. Considering metastable level populations to be dynamic and excited levels to be quasi-static, one can form population equations for each $N_{\rho}^{(z)}$ and $N_{i}^{(z)}$ in terms of these matrix elements as

$$
\begin{aligned}
\frac{\mathrm{d} N_{\rho}^{(z)}}{\mathrm{d} t} & =C_{\rho \sigma} N_{\sigma}^{(z)}+C_{\rho j} N_{j}^{(z)}+C_{\rho \sigma+} N_{\sigma+}^{(z+1)}+C_{\rho \sigma-} N_{\sigma-}^{(z-1)} \\
0 & =C_{i \sigma} N_{\sigma}^{(z)}+C_{i j} N_{j}^{(z)}+C_{i \sigma+} N_{\sigma+}^{(z+1)}+C_{i \sigma-} N_{\sigma-}^{(z-1)}
\end{aligned}
$$

Elimination of the excited populations via the quasi-static assumption leads to the collisional-radiative coefficients, which couple metastables of the ionisation stage of interest to adjacent stages and are the basis of our solution.

There is no significantly populated metastable in the case of Li-like ions, but the metastable resolved picture matters when considering recombination and ionization to and from the He-like and Be-like stages. The variation due to the inclusion of dielectronic recombination is particularly pronounced in the collisional-radiative recombination coefficient. The density dependence of this process makes it of interest here. Collisional ionization out of excited states suppresses the effect of dielectronic recombination at higher density.

The generalized collisional-radiative coefficients are computed within the Atomic Data and Analysis Structure (ADAS, Summers 2004) framework. ADAS is a collection of fundamental and derived atomic data, and codes that manipulate them. The data are organized in a form that allows generation of the collisional-radiative matrix, which is manipulated to form the derived effective recombination and ionization coefficients.

Starting with the equation for a spectral line, we have the intensity from upper level $j$ to lower level $i$, integrated over path length $h$, given by

$I_{j \rightarrow i}=h v_{j \rightarrow i} \int A_{j \rightarrow i} N_{j} \mathrm{~d} h$

where $N_{j}$ denotes the population density of ions in the upper state $j$ and $A_{j \rightarrow i}$ is the radiative transition probability.
Table 1. The enhancement factor for the intensities of four lines from Li-like ions relative to the low-density value at $N_{\mathrm{e}}=10^{6} \mathrm{~cm}^{-3}$.

\begin{tabular}{lcccc}
\hline \hline Line & $N_{\mathrm{e}}=10^{6}$ & $N_{\mathrm{e}}=10^{9}$ & $N_{\mathrm{e}}=10^{11}$ & $N_{\mathrm{e}}=10^{12}$ \\
\hline C IV 1548 & 1.00 & 1.59 & 2.93 & 3.45 \\
N v 1238 & 1.00 & 1.17 & 1.38 & 1.37 \\
O VI 1032 & 1.00 & 1.28 & 1.52 & 1.59 \\
Ne VIII 770 & 1.00 & 1.14 & 1.27 & 1.30 \\
\hline
\end{tabular}

Using the collisional-radiative matrix as described by Eqs. (1) and (2), and making the substitution

$A_{j \rightarrow i} N_{j}=\frac{N_{\text {tot }}}{N_{\mathrm{H}}} N_{\mathrm{e}}^{2} G\left(T_{\mathrm{e}}, N_{\mathrm{e}}\right)$

one can determine $G\left(T_{\mathrm{e}}, N_{\mathrm{e}}\right)$ from the populations and transition probabilities. $G\left(T_{\mathrm{e}}, N_{\mathrm{e}}\right)$ is known as the density dependent contribution function and corresponds to the well-known $G\left(T_{\mathrm{e}}\right)$ function (see Lanzafame et al. 2002). It includes the equilibrium ionisation balance, which in this case is density-dependent metastable resolved. These were calculated for four lines from Li-like ions, namely, C IV $1548 \AA$, N v $1238 \AA$, O VI $1032 \AA$ and Ne VIII $770 \AA$, the results of which are given in the next section.

\section{Results}

\subsection{Contribution function}

Four values of the electron density; $10^{6} \mathrm{~cm}^{-3}$ for the low density limit, and $10^{9} \mathrm{~cm}^{-3}$ for a typical quiet Sun electron density, $10^{11} \mathrm{~cm}^{-3}$ an active region and $10^{12} \mathrm{~cm}^{-3}$ for a flare were used with the results shown in Fig. 1. For these plots we used a temperature spacing og $\log T_{\mathrm{e}}=0.1$. Here we clearly see that with increasing $N_{\mathrm{e}}$, the $G\left(T_{\mathrm{e}}, N_{\mathrm{e}}\right)$ function shows a significant increase. Furthermore, all the lines have their peak contribution shifted to lower temperature. Folding in the Raymond \& Doyle (1981) DEM, we obtain for C IV $1548 \AA$, a $60 \%$ enhancement for $N_{\mathrm{e}}=10^{9} \mathrm{~cm}^{-3}$, leading to over a factor of three enhancement for $N_{\mathrm{e}}=10^{12} \mathrm{~cm}^{-3}$. The increase is lower for the higher temperature lines, but are still in the range of 30 to $60 \%$ larger.

\subsection{Radiative loss function}

With exception of results by Landi \& Landini (1999), all other published radiative loss functions were calculated in the low-density limit. From the above population solution, the total radiative power function is calculated as

$P_{\mathrm{rad}}=\sum_{z=0}^{z_{0}} \sum_{\rho=1}^{M_{z}}\left[P_{\mathrm{LT}, \rho}^{(z)}+P_{\mathrm{RB}, \rho}^{(z)}+\left(N_{\mathrm{H}} / N_{\mathrm{e}}\right) P_{\mathrm{RC}, \rho}^{(z)}\right]\left(N_{\rho}^{(z)} / N_{\mathrm{tot}}\right)$

with contributions arising from low level line power $\left(P_{\mathrm{LT}}\right)$, recombination-bremsstrahlung-cascade power $\left(P_{\mathrm{RB}}\right)$ and charge exchange recombination power $\left(P_{\mathrm{RC}}\right)$. In Fig. 2 , we show the percentage difference

$D i f f=\frac{P_{\text {rad }}\left(T_{\mathrm{e}}, N_{\mathrm{e}}=10^{6}\right)-P_{\text {rad }}\left(T_{\mathrm{e}}, N_{\mathrm{e}}^{i}\right)}{P_{\text {rad }}\left(T_{\mathrm{e}}, N_{\mathrm{e}}=10^{6}\right)}$ 

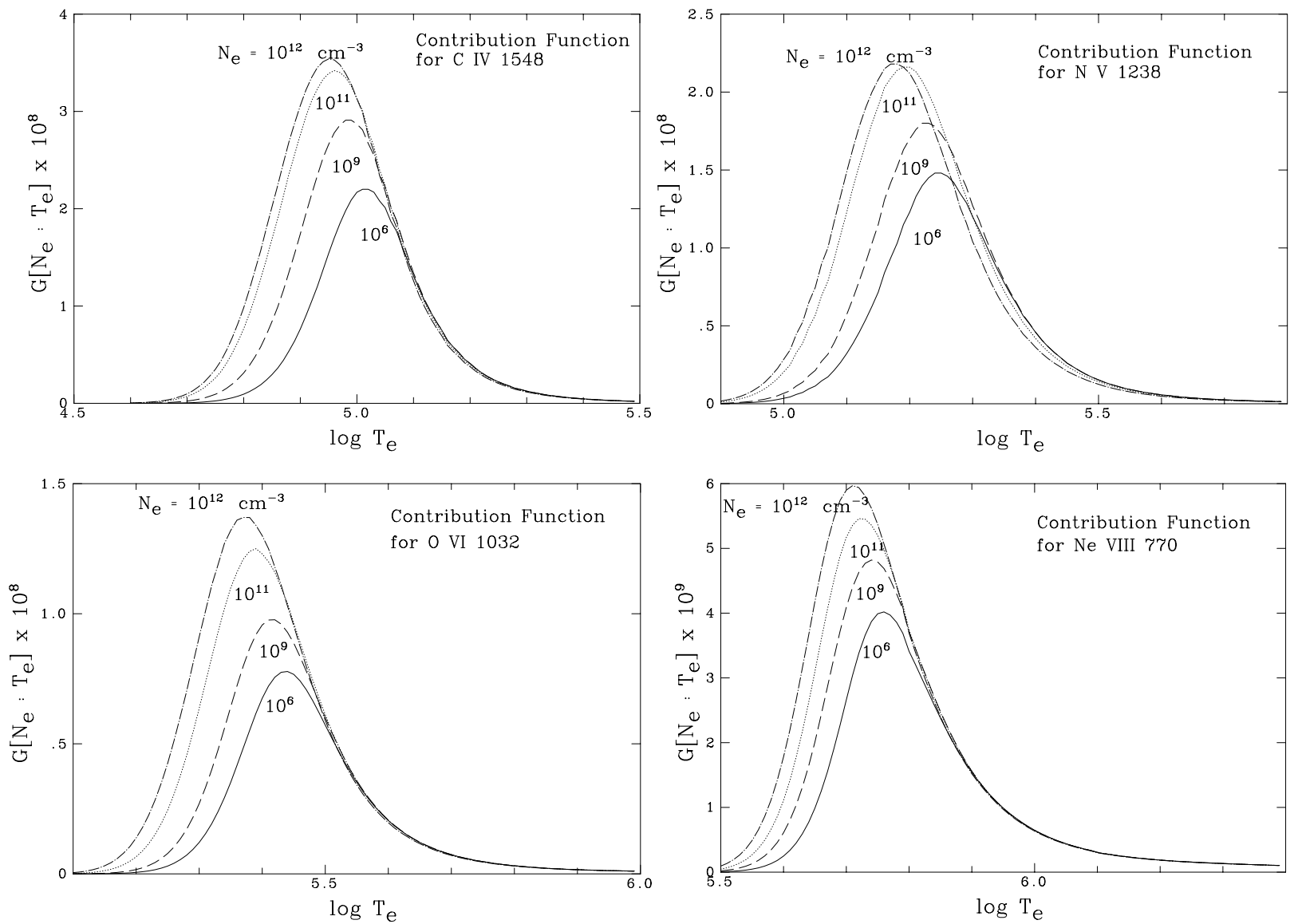

Fig. 1. The contribution function for C IV $1548 \AA$, N v $1238 \AA$, O VI $1032 \AA$ and Ne VIII $770 \AA$ A line in units of $\mathrm{cm}^{3} \mathrm{~s}^{-1}$ for four values of the electron density, $10^{6} \mathrm{~cm}^{-3}, 10^{9} \mathrm{~cm}^{-3}, 10^{11} \mathrm{~cm}^{-3}$ and $10^{12} \mathrm{~cm}^{-3}$.

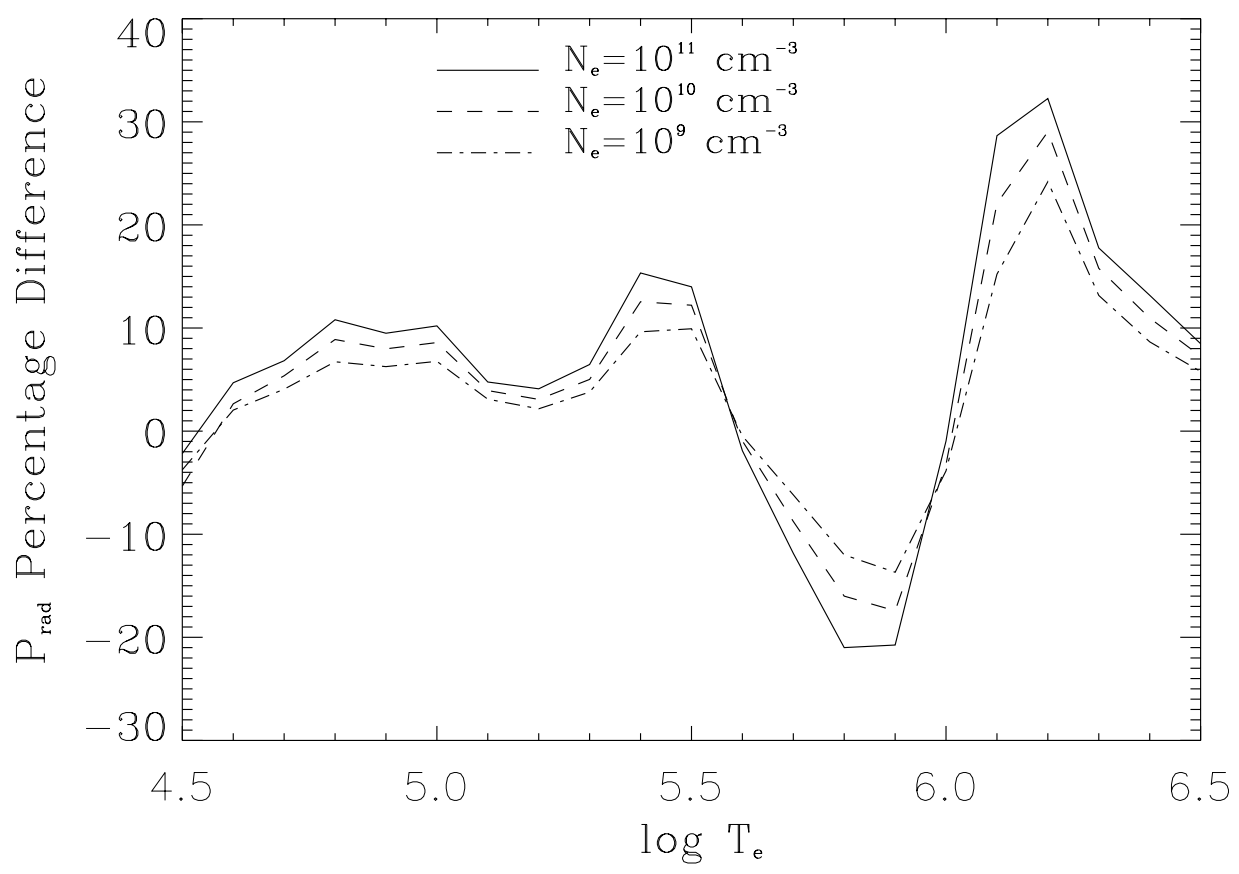

Fig. 2. The percentage difference in the radiative loss function assuming different electron densities: $10^{6}$ versus $10^{11} \mathrm{~cm}^{-3}$ (solid line), $10^{6}$ versus $10^{10} \mathrm{~cm}^{-3}$ (dashed line), $10^{6}$ versus $10^{9} \mathrm{~cm}^{-3}$ (dot-dash line). 
where $N_{\mathrm{e}}^{i}=10^{9}, 10^{10}, 10^{11} \mathrm{~cm}^{-3}$. In the transition region the difference is $10-20 \%$ while above $10^{6} \mathrm{~K}$ the difference is around $30 \%$. Elements included in the calculation were $\mathrm{H}$, $\mathrm{He}, \mathrm{C}, \mathrm{N}, \mathrm{O}, \mathrm{Ne}, \mathrm{Si}, \mathrm{S}, \mathrm{Ar}, \mathrm{Fe}$ and Ni, i.e. the most abundant elements at these temperatures. Although the detail in Fig. 2 is slightly different from that of Landi \& Landini (1999), the general result is similar. However, these differences are small and well within the errors in the atomic data. As pointed out by Landi \& Landini, larger differences can arise from an incorrect treatment of the level populations, or the use of different ionization fractions. However, the largest variation is due to abundance.

\section{Discussion}

The DEM analysis has been an important tool in the study of solar and stellar plasma over the past few decades. However, such an analysis can be suspect if the selection of lines are affected by opacity, inaccurate atomic coefficients (Lanzafame et al. 2002), or inappropriate assumptions concerning the calculation of the ionization fractions (Vernazza \& Raymond 1979). A general DEM-rule is that only resonance-type lines (therefore eliminating the electron density dependence) should be used, and since lines from Li-like ions are strong resonance lines these have been used by many authors. This however ignores the density dependence resulting from adjacent ionization stages.

Doyle \& Raymond (1983) noted that N V and, to a lesser extent, O VI implied much larger values for the emission measure during the early stages of a large solar flare. For $\mathrm{N} \mathrm{V}$ this amounted to a factor of 2 to 3 . The present results goes a long way to correcting this with perhaps the remaining difference being due to temporal variability (i.e. there was a 2 min time difference between the observation of the $\mathrm{N} v$ and the $\mathrm{O} v$ lines used in that particular study). From the results presented in the previous section, assuming that the low-density limit applies to Li-like ions leads to errors of up to factors of 2-3. It is therefore clear that these lines should not be used unless the ionization balance is treated with the necessary physics.

The inclusion of the density dependence in the total power loss has some effect, particularly in the upper transition region, although the difference is less than $20 \%$ compared to the low-density approximation.

Acknowledgements. Research at the Armagh Observatory is grantaided by the N. Ireland Dept. of Culture, Arts and Leisure. This work was funded in part by a PRTLI research grant for Grid-enabled Computational Physics of Natural Phenomena (Cosmogrid), plus PPARC Visitors grant PPA/V/S/2003/00049.

\section{References}

Bates, D. R., Kingston, A. E., \& McWhirter, R. W. P. 1962, Proc. Roy. Soc. A, 267, 297

Burgess, A., \& Summers, H. P. 1969, ApJ, 157, 1007

Del Zanna, G., Landini, M., \& Mason, H. E. 2002, A\&A, 385, 968

Doyle, J. G., \& Raymond 1984, Sol. Phys., 90, 97

Landi, E., \& Landini, M. 1999, A\&A, 347, 401

Lanzafame, A. C., Brooks, D. H., Lang, J., et al. 2002, A\&A, 384, 242

Raymond, J. C., \& Doyle, J. G. 1981, ApJ, 245, 1141

Summers, H. P., \& Hooper, M. B. 1983, Plasma Physics, 25, 1311

Summers, H. P. 2004, The ADAS User Manual, version 2.6, http://adas.phys.strath.ac.uk

Vernazza, J. E., \& Raymond, J. C. 1979, ApJ, 228, L89

Young, P. R., Del Zanna, G., Landi, E., et al. 2003, ApJS, 144, 135 